\title{
DWT based Off-line Signature Verification using Angular Features
}

\author{
Prashanth C R \\ Department of ECE, Vemana \\ Institute of Technology \\ Bangalore, India
}

\author{
K B Raja, Venugopal K R \\ Department of CSE, University \\ Visvesvaraya College of \\ Engineering, Bangalore, India
}

\author{
L M Patnaik \\ Honorary Professor \\ Indian Institute of Science \\ Bangalore, India
}

\begin{abstract}
The signature verification system is always the most sought after biometric verification system. Being a behavioral biometric trait which can be imitated, the researcher faces a challenge in designing such a system to counter intrapersonal and interpersonal variations. This papers presents DWT based Off-line Signature Verification using Angular Features (DOSVAF). The signature is resized and Discrete Wavelet Transform (DWT) is applied to get four bands. The approximation band is considered and skeletonized. The exact signature area is cropped and resized so that the fair comparison is made among the signatures to produce better result. The angular features are extracted by dividing the signature image into number of blocks. The angular features of database and test signature are compared using distance metric. It is found that the values of FAR and FRR at optimal threshold are better compared to that of existing methods.
\end{abstract}

\section{General Terms}

Pattern Recognition, Security.

\section{Keywords}

Angular features, Biometrics, Random forgery, Image Splitting, Centre of Signature.

\section{INTRODUCTION}

The personal identification is a very important aspect of security. The identification techniques opted vary according to circumstances and requirements. The traditional identification may be carried out by identity cards, pin codes, smart cards etc., but these are easily lost and forgotten [1]. A better way of individual identification is based on biometrics. The biometric verification involves identification of individuals based on physiological and behavioral features. The physiological features include iris, face, finger print, DNA etc, and the behavioral features include voice, signature, gait which are unique to a person. Since many physiological and behavioral characteristics are unique to an individual, biometrics provides a more reliable system of authentication than traditional systems. Physiological traits are the stable physical characteristics. Behavioral characteristics can change over time, but it is less expensive and less dangerous to users.

Signatures have been a primary method of identification of a person in all fields for purposes such as the credit cards, contract agreements, cheques, wills, and other important documents. Thus a signature is widely used behavioral biometric for identifying a person. In day to day life millions of signatures need to be verified, this tends to be impossible by visual inspection, therefore an automated system is necessary for determining the authenticity of the signature. Several decades have witnessed intense research in the field of signature verification, especially in the off-line signature verification.

There are two categories in signature verification based on the acquisition of the signature viz., on-line and off-line verification systems. The on-line verification process is conducted using pen and an E-pad which gives information about velocity, stroke order, acceleration, pen pressure etc., off-line process uses a static image of the signature. The offline signature verification problem is more challenging than the on-line signature verification, because the features are extracted from the static 2D image of the signature.

There is a need to develop efficient signature verification system for authenticating an individual successfully. The processing of signature strokes is difficult due to highly stylish and unconventional writing styles. The nature and the variety of the writing pen may also affect the nature of the signature obtained. The non-repetitive nature of variation of the signatures, because of age, illness, mood, stress levels, geographic location and perhaps to some extent the emotional state of the person, accentuates the problem. All these coupled together cause large intra-personal signature variation. A robust system has to be designed which should not only be able to consider these factors but also detect various types of forgeries. The off-line signature verification system shall have an acceptable trade-off between a low False Acceptance Rate (FAR) and a low False Rejection Rate (FRR) [2].

The complete signature verification is split into four stages: signature acquisition, preprocessing, feature extraction and matching. The signature acquisition step captures the images of signatures. Preprocessing step involves removal of noise and skeletonization for making the acquired signature suitable for feature extraction. Then the preprocessed image is used to extract relevant features that can distinguish signatures of different persons. The extracted features are used to verify a given signature image.

Contribution: In this paper, a technique for personal identity authentication by DWT based signature verification is proposed. The Discrete Wavelet Transform is used for enhancing the spatial domain features and reduces noise. The angular features are extracted with respect to centre of the signature. Matching between the test image and the database image is done by setting a threshold point.

Organization of the paper: The rest of the paper is organized as follows. In section 2, related work is discussed. In section 3 , the proposed signature verification model is discussed. In section 4, the DOSVAF algorithm is presented. The performance analysis is presented in section 5 and concluded in section 6 . 


\section{LITERATURE SURVEY}

Ramachandra et al., [3] proposed Robust Off-line Signature Verification based on Global Features (ROSVGF) for skilled and random forgeries. The model extracts the features which are preprocessed by normalization, binarization and thinning. The feature extraction technique consists of global features such as aspect ratio, maximum horizontal histogram and maximum vertical histogram, horizontal and vertical centre of signature and signature area. Sabourinand and Drouhard [4] proposed Automatic Handwritten Signature Verification System (AHVS), which can cope up with all types of forgeries. In order to eliminate rapidly gross forgery directional Probability Density Function (PDF) is chosen as a global feature vector. The comparison of features is based on Neural Network using classical back propagation algorithm.

Stephane Armand et al., [5] proposed an effective method using unique structural features extracted from signature contour. A novel combination of Modified Direction Feature (MDF) and additional distinguishing features such as centroid, surface area, length and skew are used for classification. A Resilient Backpropagation (RBP) neural network and Radial Basis Function (RBF) network are compared in terms of accuracy.

Luiz Oliveira et al., [6] proposed a writer independent model which reduces the pattern recognition problem to a 2-class problem, hence, makes it possible to build robust signature verification system even when few signatures per writer are available. Receiver Operating Characteristics (ROC) curves are used to improve the performance of the system. The impacts of fusion strategies to combine the partial decisions are yielded by SVM classifiers.

Taylan Das and Canan Dulger [7] proposed a technique based on Neural Network (NN) approach trained with particle swarm optimization (PSO) algorithm using grid segmentation model. The image is scanned and noise is removed by normalization, area cutting, resampling, erode dialate and thinning. To test the performance of the proposed PSO-NN algorithm three types of forgeries i.e., random, skilled and unskilled are examined.

Azzopardi and Camilleri [8] proposed Radial Basis Function Neural Networks (RBFNN) for Off-line Signature Verification. The signature database was collected using intrapersonal variations for evaluation. Global, grid and texture features are used as feature sets. The system is extensively tested with random forgeries and high recognition rates obtained demonstrate the effectiveness of the architecture. The best results are obtained using combined feature vector.

Ghandali and Moghaddam [9] proposed a model based on Image Registration, Discrete Wavelet Transform (DWT) and Image Fusion. Training signatures of each person are registered to overcome shift and scale problems. To extract features DWT is used, then several registered instances of each signature are fused together to generate reference pattern of signatures. In the classification phase Euclidean Distance is used.

Milena Souza et al., [10] proposed a model where five distances are calculated and evaluated using the database. They are furtherest, nearest, template, central and ' $n$ 'central. In normalization phase each distance scale invariant is established. These calculated distances are used as an input vector for an Auto Encoder Neural Network.
Lajish [11] presented a feature extraction method for off-line recognition of segmented Malayalam handwritten characters for their gray scale images without the usual step of binarization. Debasish Jena et al., [12] proposed a Off-line Signature Verification system which is based on selecting 60 feature points. The classification of the feature points utilize statistical parameters like mean and variance. It takes care of skilled and unskilled forgeries and the evaluation is based on FRR (False Rejection Ratio) and FAR (False Acceptance Ratio).

Cesar Santos et al., [13] proposed off-line signature verification based on graphometric features and neural network classifiers. The number of signature samples required to train the classifiers like Neural Networks (NN), Hidden Markov Model (HMM) and Support Vector Machine (SVM) is reduced. Heng Ma et al., [14] proposed an approach to resolve the Off-line Chinese signature verification problem using radial segmentation method. The features of signature are obtained by finding the principle axis of the signature and equally dividing the signature into a number of radial regions. The Euclidian distance is for comparing the extracted features with the threshold. Mustafa Agil Muhammed Balbed et al., [15] presented Hidden Markov Model (HMM) based off-line verification system to distinguish between genuine and forged signatures. The four features extracted include pixel density, centre of gravity, distance and angle. The performance is evaluated based on the Analysis of Variance.

$\mathrm{Vu}$ Nguyen et al., [16] proposed a method using global features based on the boundary of a signature and its projection. The first global feature is derived from total energy a writer uses to create signature. The second feature employs information from vertical and horizontal projection of a signature, distance between keystrokes in image and height width of signature. The combination of these features along with Modified Direction Feature and Support Vector Machine is used. Wan-Suck Lee et al., [17] described a off-line signature verification technique based on dynamic time warping. The signature verification performance is evaluated using neural network.

Jesus Vargas et al., [18] proposed the representation of the information from a static image of handwritten signature to analyze off-line signature verification. The histogram is calculated using pseudo-cepstral coefficients. The unique minimum phase sequence is estimated and is used as feature vector for signature verification. Hai Rong Lv et al., [19] proposed Hidden Markov Model approach for off-line Signature Verification System. The images are represented as a landmark point set which includes turning points, isolated points, trifurcate points, intersection points and termination points. A grid partition technique used shows remarkable improvement. Muhammad Reza Pourshahabi et al., [20] proposed a off-line Handwritten Signature Identification and Verification system based on Contourlet transform and is used as feature extractor for system. The image is enhanced by removing noise and normalized. The Contourlet coefficients computed forms feature vector and Euclidean distance is used for matching.

Sharifah Mumtazah Syed Ahmad et al., [21] proposed an Offline Signature Verification system that is built using statistical techniques. The Hidden Markov Model is used to build a reference model for each local feature extracted for a particular user. The verification phase involves statistical techniques. Bharadi and Kekre [22] explained Off-line Signature Recognition Systems using normalization, noise removal and thinning of the signature. The features extracted 
include global features, Walsh coefficient of pixel distribution, codeword histogram based on clustering technique, Grid features and Successive Geometric centers.

\section{DOSVAF MODEL}

In this section, definitions and block diagram of DOSVAF system are explained.

\subsection{Definitions}

i) Signature: It is the handwritten depiction of a person's name.

ii) Random Forgery: It is a signature done by a person, who does not know the shape and structure of genuine signature.

iii) Casual Forgery: It is a forgery signature produced with the knowledge about the genuine writer's name only.

iv) Skilled forgery: Forgeries which are produced by a professional imposter or person who has experience in copying.

v) False Accept Rate (FAR): It is the total number of forgery signatures accepted by the system with respect to the total number of comparisons made.

vi) False Rejection Rate (FRR): It is the total number of genuine signatures rejected by the system with respect to the total number of comparisons made.

vii) Equal Error Rate (EER): It is the threshold value for which the FAR and FRR values are equal. A smaller EER indicates a better performance.

\subsection{DOSVAF System}

Figure 1 gives the block diagram of DOSVAF System, which verifies the authenticity of given signature of a person. The signature images for study are collected from the different subjects to create the database. The DWT is used for improving the spatial domain features of the signatures and to reduce the noise. The Angular features are extracted with respect to centre of the signature by vertical and horizontal splitting. The extracted features are compared using distance metric.

\subsubsection{Image Acquisition}

Signatures are obtained from subjects on a blank paper at different timings depending upon the mood and stress levels and they are scanned to get images of 960 dpi resolution in bmp format. This forms the database for the work. The genuine signature is forged after sufficient training. A sample genuine signature so acquired is as shown in Figure 2.

\subsubsection{Preprocessing}

The acquired signatures of each person have different sizes. The signature is resized to $300 * 900$ and DWT is applied to get approximation band with low frequency components. The approximation band of size $150 * 450$ is considered as it has significant information.

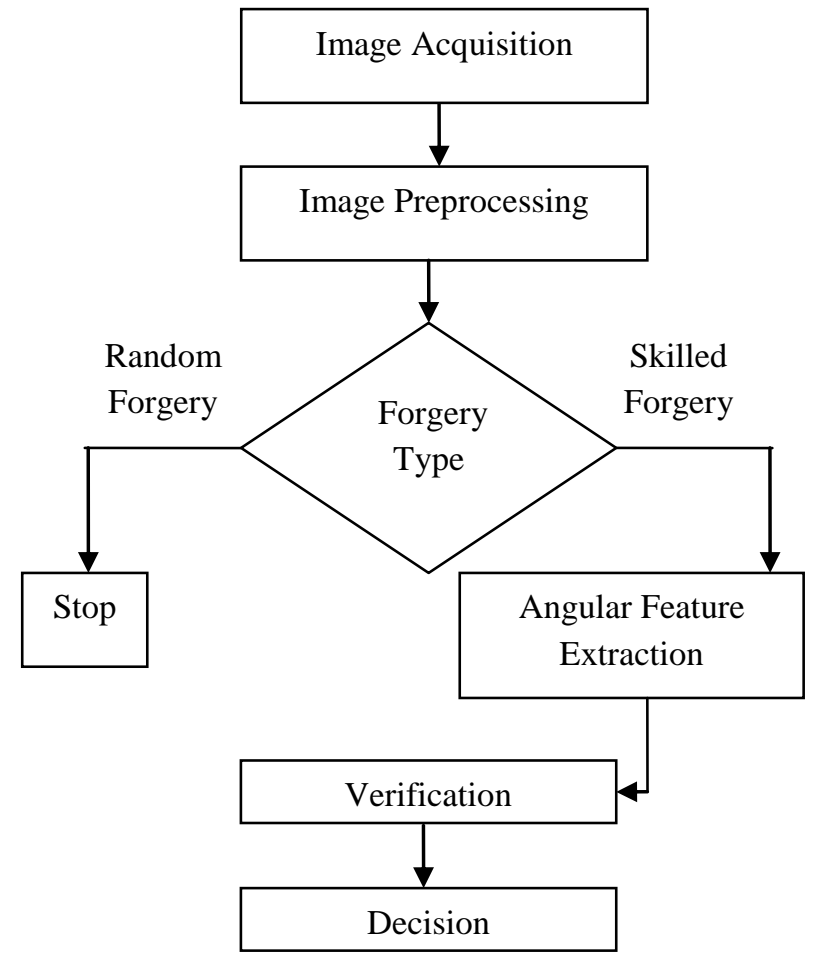

Fig 1: Block diagram of DOSVAF system

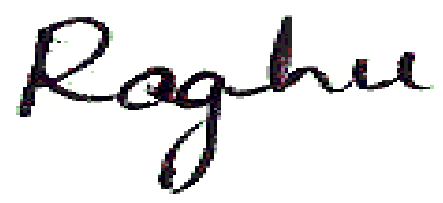

Fig 2: Sample Genuine Signature

\subsubsection{Skeletonization}

Reducing image to its single pixel width is called skeletonization. A binary 2D object can be represented by its skeleton, which consists of the spines of the object parts. A 2D skeleton may consist of curves (spines), isolated 2D points and possibly, 2D surfaces. The generation of a skeleton is realized by applying an iterative process which erodes the object layer by layer until only the object spines remain, which form the skeleton of the $2 \mathrm{D}$ object. The skeletonization is done by removing the pixels on the boundary of the signature image without allowing the signature image to break apart. This process is achieved by performing morphological operations on signature image using skel function. The Figure 3 shows the signature after skeletonization.

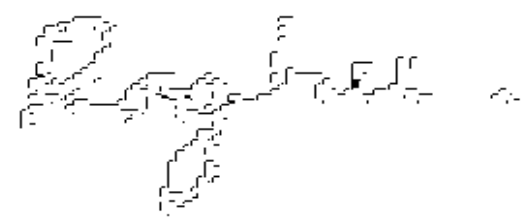

Fig 3: Signature after Skeletonization 


\subsubsection{Exact Signature Area}

The signature image might not be present on the entire frame. So, the exact signature area is considered in the skeletonized image for further analysis. This reduces verification time and is cost effective. The signature image is scanned from top to bottom to get the first row (black pixel) $a 1$ of the image and the last row (black pixel) $a 2$ of signature. The first row $a 1$ of the exact signature area is the value of the row variable $i$ corresponding to first black pixel and last row $a 2$ is the value of the row variable $i$ corresponding to last black pixel, while scanning from top to bottom. The horizontal scanning for finding exact signature area is given by the Equation 1

$$
\sum_{i=1}^{N} \sum_{j=1}^{M} I_{s}(i, j)=0
$$

The signature image is scanned from left to right to get the first column (black pixel) $a 3$ of the image and the last column (black pixel) $a 4$ of the signature. The first column $a 3$ of the exact signature area is the value of the column variable $j$ corresponding to first black pixel and last column $a 4$ is the value of the column variable $j$ corresponding to last black pixel, while scanning from left to right. The horizontal and vertical scanning for finding exact signature area is given by the Equation 2. The Figure 4 shows the image with exact signature area.

$$
\sum_{j=1}^{M} \sum_{i=1}^{N} I_{s}(i, j)=0
$$

where $I_{s}(i . j)$ is the skeletonized signature image.

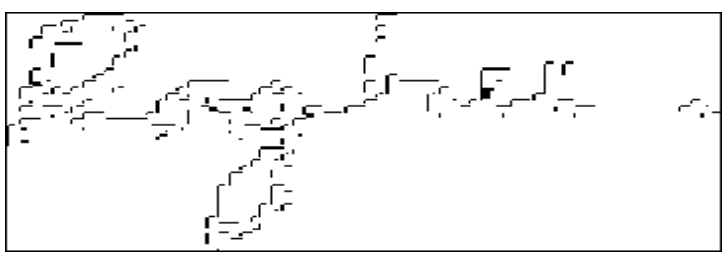

Fig 4: Exact Signature Area

\subsubsection{Random Forgery Detection}

To detect Random Forgery at the early stage, the number of rows and columns are obtained for all the ten genuine signatures. The average number of rows and columns are obtained to be compared with those of the test signature. The difference between the average number of rows and columns and those of the test signature is computed. If the difference falls in the predefined threshold then the test signature is proceeded for angular feature extraction stage else the random forgery is detected and the verification process is aborted.

The signature image is scanned horizontally from the top to get beginning row $R_{l}$ of the signature and it is scanned horizontally from bottom to get last row $R_{2}$ of the signature. The difference $r$ between top row $R_{1}$ and bottom row $R_{2}$ gives the number of rows representing the signature image and is given by the Equation 3. Similarly the number of columns $c$ representing the signature image is obtained and is given by the Equation 4.

$$
\begin{aligned}
& r=R_{1}-R_{2} \\
& c=C_{1}-C_{2}
\end{aligned}
$$

The same procedure is followed to find the number of rows and columns representing the all the signatures of a person. The average number of rows $R_{\text {avg }}$ and average number of columns $C_{\text {avg }}$ are calculated using the Equations 5 and 6.

$$
R_{\text {avg }}=\frac{\sum_{i=1}^{N_{G S}} r_{i}}{N_{G S}}
$$

Where $r_{i}$ is the number of rows representing the $i^{\text {th }}$ signature and $N_{G S}$ is the total number of genuine signatures.

$$
C_{\text {avg }}=\frac{\sum_{j=1}^{N_{G S}} c_{j}}{N_{G S}}
$$

Where $C_{j}$ is the number of columns representing the $j^{\text {th }}$ signature and $N_{G S}$ is the total number of genuine signatures.

If the absolute value of the difference between $R_{a v g}$ and number of rows $R_{T i}$ representing the test signature is less than or equal to 5 and If the absolute value of the difference between $C_{a v g}$ and number of columns $C_{T i}$ representing the test signature is less than or equal to 10 , then the angular feature extraction and verification are carried out on the test signature else random forgery is detected and verification process is aborted.

\subsubsection{Feature Extraction}

The preprocessed signature image is made to undergo vertical splitting and horizontal splitting. The skeleton of the signature image is scanned from left to right and top to bottom to calculate the total number of black pixels and is given by the Equations 7 and 8 . Then the image is divided into two halves with respect to the number of black pixels by two lines, vertically and horizontally which intersects at a point called the geometric centre.

$$
\begin{aligned}
& \sum_{x=1}^{N} \sum_{y=1}^{M} I_{\text {exact }}(x, y)=0 \\
& \sum_{y=1}^{M} \sum_{x=1}^{N} I_{\text {exact }}(x, y)=0
\end{aligned}
$$

The procedure for finding angular feature points by horizontal and vertical splitting is explained as follows. The signature image is split with horizontal line passing through geometric centre of image to get top and bottom parts of image. The coordinates of the boundary of each part or block is stored. 
The geometric centre for each block is obtained by locating a point where number of black pixels is half of the total number of black pixels in the block. Geometric centre is found for top and bottom blocks respectively. The top block is split with a vertical line to find the geometric centre for left and right parts of top block. Again the coordinates of the boundary of each block is obtained. Similarly, the bottom block is split with a vertical line to find the geometric centre for left and right parts of bottom block. Then, each part is again split through their geometric centre to obtain angular feature points as shown in Figure 5 .

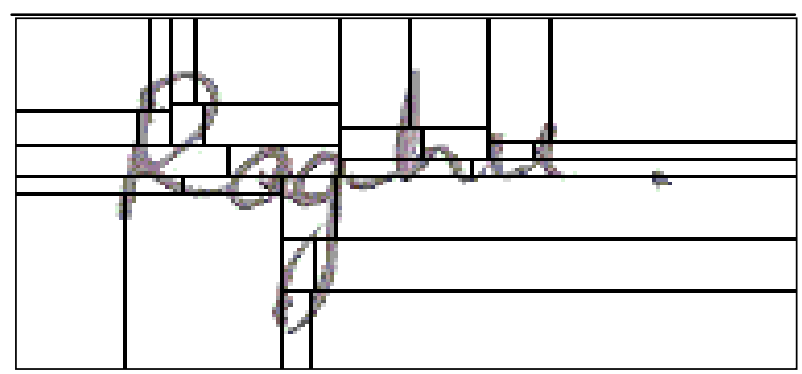

Fig 5: Horizontal and Vertical splitting of the signature

The process of vertical and horizontal splitting of signature leads to 128 blocks. The centre of signature is obtained for these blocks which are result of image splitting. The angular features for each of these blocks are obtained. The procedure of obtaining these features is explained as follows. The top left corner of the original image is always considered as the reference point. The centre of signature obtained by applying the earlier procedure is taken as the other point. By using the distance formula given by Equation 9, the distance between the reference point and centre of signature is calculated.

$$
\text { Distance }=\sqrt{\left(i_{2}-i_{1}\right)^{2}+\left(j_{2}-j_{1}\right)^{2}}
$$

where $\left(i_{2}, j_{2}\right)$ and $\left(i_{1}, j_{1}\right)$ are the coordinates of two points.

Consider the reference point, $A(1,1)$ and the centre $C(i, j)$ of signature as shown in the Figure 6 . The angle $\theta$ is computed using the Equation 10.

$$
\theta_{i}=\cos ^{-1}\left(\frac{A B}{A C}\right)
$$

where $i=1,2,3,4 \ldots \ldots . .128$, adjacent side $A B$ and hypotenuse $A C$ are given by Equations 11 and 12 respectively.

$$
\begin{aligned}
& A C=\sqrt{(i-1)^{2}+(j-1)^{2}} \\
& A B=\sqrt{(j-1)^{2}}
\end{aligned}
$$

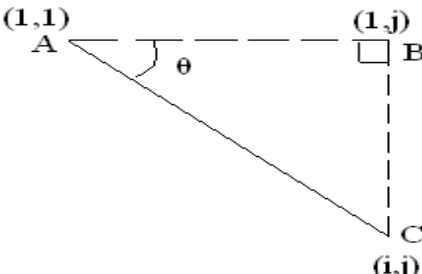

Fig 6: Measurement of angular feature

The right angled triangle $\mathrm{ABC}$ is considered and the cosine of the angle is obtained. This is done by using the concept of trigonometry. We apply the distance formula as given in Equation 11 and Equation 12 to obtain the hypotenuse and the adjacent side respectively. The cosine of the angle is obtained by taking the ratio of adjacent side $\mathrm{AB}$ and the hypotenuse AC. This mathematical computation is obtained by using the matlab command acos which computes the cosine in radians. Radians are preferred over angles as the angles are found to have large variations which make the comparison difficult. The same procedure is carried out for all the blocks and the angles are stored in an array.

The number of blocks can be increased further but there may be the case where the angle computed would be infinity. As the pixel appears on the top left corner of the block, hypotenuse becomes zero. So the numbers of blocks are limited to 128. This completes first phase of the feature extraction.

The second phase consists of division of image into dimensions which are predefined. The image is divided into ten square blocks containing 1, 4, 9, 16, 25, 36, 49, 64, 81 and 100 pixels as shown in the Figure 7. In Figure 7, a, b, c and d correspond to division of the signature image from top left, top right, bottom left and bottom right respectively. Each division produces 10 blocks. The procedure is repeated for the remaining 3 parts to get 30 more blocks. This leads to totally 40 blocks. The angular features for these 40 blocks are found using the same procedure as explained before. This completes the extraction of 168 angular features from the preprocessed signature image.

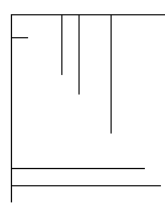

b

$\mathrm{c}$

d

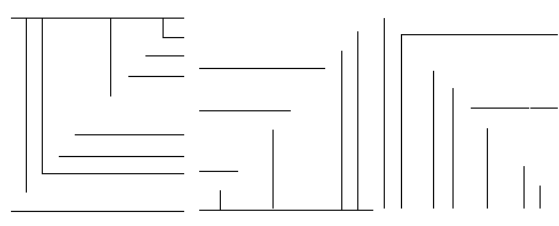

\section{Fig 7: Division of signature image}

\subsubsection{Verification}

The comparison between the genuine signature and test signature is made by computing the difference between the angles in radians obtained for both the signatures. A threshold is set which decides the authenticity of the signature.

The threshold coefficient value of 0.26 radians is considered for verification purpose. The absolute difference between $i^{\text {th }}$ angle $A G i$ of the genuine signature and $i^{\text {th }}$ angle ATi of the test signature is compared with the threshold coefficient of 0.26 radians and is given by the Equation 13 .

$$
\left|\left(A_{G i}-A_{T i}\right)\right| \leq 0.26
$$


where $i=1,2,3,4 \ldots \ldots . .128$.

If the number of angles having difference less than or equal to 0.26 radians, is greater than or equal to 133 then it is considered genuine otherwise forged signature.

\section{DOSVAF ALGORITHM Problem definition:}

Consider a signature image of a subject whose identity has to be verified. The objectives are to

$i$. Preprocess the acquired signature image

ii Extract the angular features.

iii. Verify the authenticity of the test signature by taking the angular difference.

Assumptions:

$i$. The signature image is captured using scanner

ii.The input signature image is 8 bit gray scale image of bitmap(bmp) format, having vertical and horizontal resolution of $960 \mathrm{dpi}$

iii The minimum size of the signature image is $100 * 100$ at any stage

Table 1 shows the algorithm in which the authenticity of the test signature image is verified. Firstly, the signature is acquired using a scanner and is preprocessed to remove the noise contents and to bring it to the form suitable for feature extraction. Then, the features are extracted from the preprocessed images by vertical and horizontal splitting. These blocks are further analyzed to obtain the centre of the signature. The cosine is now obtained with respect to centre of the signature. The features of various signatures are compared with threshold and a decision is made whether the signature is genuine or forgery.

\section{Table 1. DOSVAF Algorithm}

- Input: Reference and Test signatures.

- Output: Verified signature.

1. Signature Acquisition.

2. Preprocessing to transform the signature image suitable for feature extraction.

3. Horizontal and vertical splitting is done to obtain the blocks. The centre of signature of all these blocks is computed. The cosine of the angle of the reference point with respect to centre of signature is extracted for all the blocks.

4. Extracted angular features are stored in the database.

5. The features in database are compared with the feature points extracted from the test signature using the same concept

6. The threshold value is fixed at 0.26 radians. For a signature to be genuine, the difference between the two angles in verification stage should not exceed the threshold value. If the number of such angles is greater than or equal to 133 , then the signature is declared genuine otherwise forged.

\section{PERFORMANCE ANALYSIS}

For the performance analysis, the database consisting of 80 signature samples of 4 subjects is considered. Among them, 40 samples are genuine and 40 samples are forged. The
Matlab version 7.0.1 is used for implementation of the proposed algorithm.

Ten signature samples are obtained from each subject at different timings. For each subject 10 skilled forgery samples are obtained to get a total of 40 skilled forgery samples. Samples of genuine and forgery signatures used for experimentation are as shown in Figures 8 and 9 respectively.

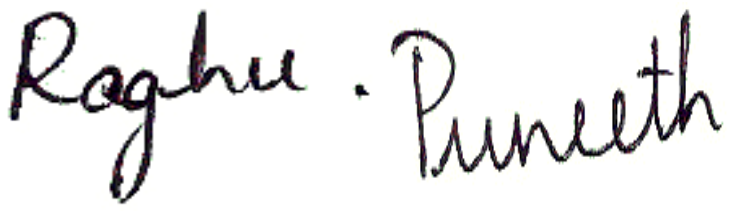

Fig 8: Sample Genuine signatures

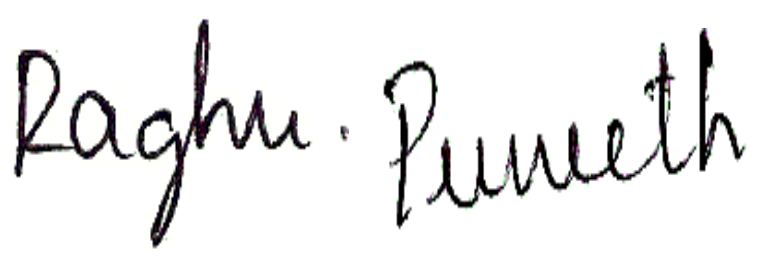

Fig 9: Sample Skilled Forgery signatures

For each subject, one out of ten genuine signature samples is taken as reference and it is compared with 9 other genuine samples. This process is continued until all signature samples are mutually compared. The same process is repeated for the signature samples of other 3 subjects also. Thus FRR is calculated. One sample of each subject is considered as reference and is compared with the 10 forged samples of the same subject. This process is repeated for all the signature samples. Thus FAR is calculated. Table 2 gives the number of signature samples taken and the results for the various signatures tested.

Table 2. FAR and FRR values

\begin{tabular}{|c|c|c|c|}
\hline Signatures & $\begin{array}{c}\text { No. of } \\
\text { samples }\end{array}$ & $\%$ FAR & $\%$ FRR \\
\hline Original & 40 & ----- & 4.995 \\
\hline Skilled Forgery & 40 & 8.500 & ----- \\
\hline
\end{tabular}

It is observed that FAR and FRR values obtained with angular difference threshold of 0.26 are less compared to that of existing methods. Table 3 shows the comparison of the proposed DOSVAF technique with the existing ROGCOSV [23] and SSCOSV [24]. FAR and FRR obtained is $8.5 \%$ and $4.995 \%$ respectively.

Table 3. Comparative analysis of FAR and FRR

\begin{tabular}{|c|c|c|c|}
\hline $\begin{array}{c}\text { Error } \\
\text { rates }\end{array}$ & $\begin{array}{c}\text { ROGCOSV } \\
{[23]}\end{array}$ & $\begin{array}{c}\text { SSCOSV } \\
{[24]}\end{array}$ & DOSVAF \\
\hline FAR & 25.11 & 8.88 & 4.995 \\
\hline FRR & 14.66 & 7.76 & 8.500 \\
\hline
\end{tabular}


Table 4 shows the variation of FAR and FRR depending on the threshold parameter taken to decide the genuineness of a signature image. As the threshold increases, the FAR increases and FRR decreases. If a high threshold is chosen then the FRR is increased, but at the same time the FAR is decreased. If a low threshold is chosen then the FAR is increased, but at the same time the FRR is decreased. If the FAR of a system is equal to the FRR then the system is said to be in an optimal state.

Table 4. Variation of FAR and FRR with Threshold

\begin{tabular}{|c|c|c|c|}
\hline Sl. No. & Threshold & \%FRR & \%FAR \\
\hline 1 & 0.10 & 100 & 0 \\
\hline 2 & 0.15 & 85.5 & 0.25 \\
\hline 3 & 0.20 & 45.52 & 0.50 \\
\hline 4 & 0.25 & 11.65 & 4.75 \\
\hline 5 & 0.26 & 4.995 & 8.50 \\
\hline 6 & 0.30 & 1.11 & 22.75 \\
\hline 7 & 0.35 & 0 & 51.25 \\
\hline 8 & 0.40 & 0 & 75.12 \\
\hline
\end{tabular}

Figure 10 shows the graph of FAR and FRR obtained for different values of threshold to compare the performance of signature verification system. As threshold increases, the value of FRR decreases whereas FAR increases. The value of EER obtained is 7.2 corresponding to optimal threshold of 0.256 at a point where FAR equals to FRR. This value of ERR is better compared to many of the existing off-line signature verification systems.

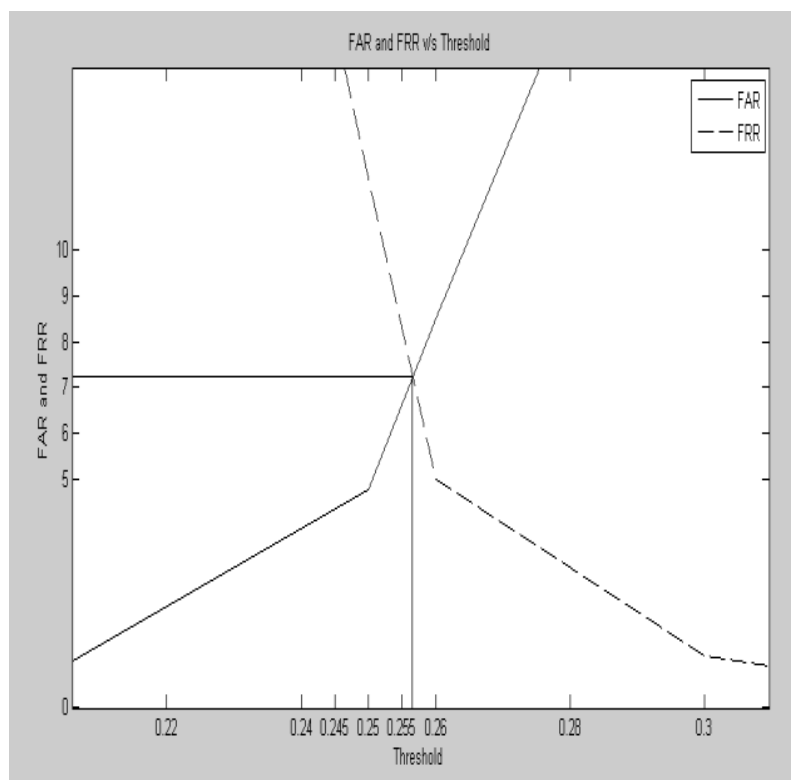

Fig 10: FAR and FRR against Threshold

\section{CONCLUSION}

The DOSVAF algorithm based on the angular features is more efficient and gives better results than the existing techniques against the skilled forgeries, because of the extraction of features in two phases. The DWT is used in the preprocessing stage, and angular features are extracted. The results are better than many other existing techniques.

Though we are able to achieve improved results, there are still high to implement in places where user authentication is the topmost concern. Hence there is scope for algorithms which give still lower error rates.

\section{REFERENCES}

[1] Anil K Jain, Arun Ross and Salil Prabhakar, "An Introduction to Biometric Recognition," IEEE Transactions on Circuits and Systems for Video Technology, vol. 14, no.1, pp. 1-29, 2004.

[2] Kresimir Delac, Mislav Grgic, "A Survey of Biometric Recognition Methods," 46th International Symposium on Electronics in Marine, pp. 184 -193, 2004.

[3] Ramachandra A C, Jyothi Srinivasa Rao, K B Raja, K R Venugopal and L M Patnaik, "Robust Off-line Signature Verification based on Global Features," IEEE International Advance Computing Conference, pp. 11731178, March 2009.

[4] Robert Sabourin and Jean-Pierre Drouhard, "Impact of Signature Legibility and Signature Type in Off-line Signature Verification," IEEE InternationalConference on Pattern Recognition, pp. 321-325, 1992.

[5] Stephane Armand, Michael Blumenstein and Vallipuram Muthukkumarasamy, "Off-line Signature Verification using the Enhanced Modified Direction Feature and Neural-based Classification," IEEE International Joint Conference on Neural Networks, pp. 684 -689, July 2006.

[6] Luiz S Oliveira, Edson Justino, and Robert Sabourin, "Off-line Signature Verification using WriterIndependent Approach," Proceedings of International Joint Conference on Neural Networks, pp. 2539-2544, August 2007.

[7] M Taylan Das and L Canan Dulger, "Off-line Signature Verification with PSO-NN Algorithm," Seventh International Conference on Intelligent Systems Design and Applications, pp. 843 - 848, 2007.

[8] George Azzopardi and Kenneth P Camilleri, "Off-line Handwritten Signature Verification using Radial Basis Function Neural Networks," IEEE International Conference on Electrical and Electronics (INDICON2008), pp. 17-22, 2008.

[9] Samaneh Ghandali and Mohsen Ebrahimi Moghaddam, "Off-line Persian Signature Identification and Verification based on Image Registration and Fusion," Journal of Multimedia, Vol. 4, No. 3, pp.137-144, June 2009.

[10] Milena R P Souza, Leandro R Almeida, and George D C Cavalcanti, "Combining Distances Through an Autoencoder Network to Verify Signatures," tenth Brazilian Symposium on Neural Networks, pp.63-72, October 2008.

[11] Lajish V L, "Handwritten Character Recognition using Gray-scale based State-Space Parameters and Class Modular Neural Networks," IEEE International Conference on Signal Processing, pp. 374-379, January 2008. 
[12] Debasish Jena, Banshidahar Majhi, and Sanjay Kumar Jena, "Improved Off-line Signature Verification Scheme using Feature Point Extraction Method," Journal of Computer Science, pp. 111-116, 2008.

[13] Cesar Santos, Edson J R Justino, Flavio Bortolozzi, and Robert Sabourin, "An Off-line Signature Verification method based on the Questioned Document Expert's Approach and a Neural Network Classifier," International Workshop on Frontiers in Handwriting Recognition, pp. 498-502, October 2004.

[14] Heng Ma, Wen-Wei Yang, and Chia-Chend Liu, "Offline Chinese-Based Signature Verification using a Threshold Self-Organizing Map," Journal of the Chinese Institute of Industrial Engineers, Vol. 24, No. 3, pp. 225235, 2007.

[15] Mustafa Agil Muhamad Balbed, Sharifah Mumtazah Syed Ahmad, and Asma Shakil, "ANOVA-Based Feature Analysis and Selection in HMM-Based Off-line Signature Verification System," International Conference on Innovative Technologies in Intelligent Systems and Industrial Applications, pp. 66-69, July 2009.

[16] Vu Nguyen, Michael Blumenstein, and Graham Leedham, "Global Features for the Off-line Signature Verification Problem," tenth International Conference on Document Analysis and Recognition, pp. 1300-1304, 2009.

[17] Wan-Suck Lee, N Mohankrishnan, and Mark J Paulik, "Improved Segmentation through Dynamic Time Warping for signature Verification using a Neural Network Classifier," International Conference on Image Processing, Vol. 2, pp. 929-933, 1998.

[18] Jesus F Vargas, Miguel A Ferrer, Carlos M Travieso, and Jesus B Alonso, "Off-line Signature Verification Based

\section{AUTHOR'S PROFILE}

Prashanth $\mathbf{C} \mathbf{R}$ received the BE degree in Electronics and the ME degree in Digital Communication from Bangalore University, Bangalore. He is pursuing his Ph.D. in Computer Science and Engineering of Bangalore University under the guidance of Dr. K. B. Raja, Assistant Professor, Department of Electronics and Communication Engineering, University Visvesvaraya College of Engineering. He is currently an Assistant Professor, Dept. of Electronics and Communication Engineering, Vemana Institute of Technology, Bangalore. His research interests include Computer Vision, Pattern Recognition, Biometrics, and Communication Engineering. $\mathrm{He}$ is a life member of Indian Society for Technical Education, New Delhi, member of IEEE and IACSIT.

K B Raja is an Assistant Professor, Dept. of Electronics and Communication Engineering, University Visvesvaraya college of Engineering, Bangalore University, Bangalore. He obtained his $\mathrm{BE}$ and $\mathrm{ME}$ in Electronics and Communication Engineering from University Visvesvaraya College of Engineering, Bangalore. He was awarded Ph.D. in Computer Science and Engineering from Bangalore University. He has over 90 research publications in refereed International Journals and Conference Proceedings. His on Psuedo-Cepstral Coefficients," tenth International Conference on Document Analysis and Recognition, pp. 126-130, 2009.

[19] Hai Rong Lv, Wen Jun Yin, and Jin Dong, "Off-line Signature Verification based on Deformable Grid Partition and Hidden Markov Models," IEEE International Conference on Multimedia and Expo, pp. 374-377, 2009.

[20] Muhammad Reza Pourshahabi, Mohamad Hoseyan Sigari, and Hamid Reza Pourreza, "Off-line Handwritten Signature Identification and Verification using Contourlet Transform," International Conference of Soft Computing and Pattern Recognition, pp. 670-673, 2009.

[21] Sharifah Mumtazah Syed Ahmad, Asma Shakil, Masyura Ahmad Faudzi, Rina Md. Anwar, and Mustafa Agil Muhamad Balbed, "A Hybrid Statistical Modelling, Normalization and Inferencing Techniques of an Off-line Signature Verification System," World Congress on Computer Science and Information Engineering, pp. 6 11, 2009.

[22] V A Bharadi and H B Kekre, "Off-line Signature Recognition Systems," International Journal of Computer Applications, Vol. 1, No. 27, pp. 61-70, 2010.

[23] H N Prakash and D S Guru, "Relative Orientations of Geometric Cntroid for off-line Signature Verification," International Conference on Advances in Pattern Recognotion, pp. 201-204, 2009.

[24] Prashanth C R, K B Raja, K R Venugopal, and L M Patnaik, "Standard Scores Correlation based Off-line Signature Verification System," International Conference on Advances in Computing, Control, and Telecommunication Technologies, pp. 49-53, 2009.

research interests include Image Processing, Biometrics, VLSI Signal Processing, computer networks

K $\mathbf{R}$ Venugopal is currently the Principal, University Visvesvaraya College of Engineering, Bangalore University, Bangalore. He obtained his Bachelor of Engineering from University Visvesvaraya College of Engineering. He received his Masters degree in Computer Science and Automation from Indian Institute of Science, Bangalore. He was awarded Ph.D. in Economics from Bangalore University and Ph.D. in Computer Science from Indian Institute of Technology, Madras. He has a distinguished academic career and has degrees in Electronics, Economics, Law, Business Finance, Public Relations, Communications, Industrial Relations, Computer Science and Journalism. He has authored 27 books on Computer Science and Economics, which include Petrodollar and the World Economy, C Aptitude, Mastering C, Microprocessor Programming, Mastering C++ etc. He has been serving as the Professor and Chairman, Department of Computer Science and Engineering, University Visvesvaraya College of Engineering, Bangalore University, Bangalore. During his three decades of service at UVCE he has over 275 research papers to his credit. His research interests include computer networks, parallel and distributed systems, digital signal processing and data mining. 
L M Patnaik is the Honorary Professor, Indian Institute of Science, Bangalore, India. During the past 35 years of his service at the Indian Institute of Science, Bangalore, He has over 550 research publications in refereed International Journals and Conference Proceedings. He is a Fellow of all the four leading Science and Engineering Academies in India; Fellow of the IEEE and the Academy of Science for the Developing World. He has received twenty national and international awards; notable among them is the IEEE
Technical Achievement Award for his significant contributions to high performance computing and soft computing. His areas of research interest have been parallel and distributed computing, mobile computing, CAD for VLSI circuits, soft computing, and computational neuroscience. 